\title{
Melanoma Surgery: Why Don't We Let the Guidelines Guide Practice?
}

\author{
Ravinder Kang, MD, MS and Sandra L. Wong, MD, MS \\ Department of Surgery, The Geisel School of Medicine at Dartmouth, The Dartmouth Institute for Health Policy and \\ Clinical Practice, Dartmouth-Hitchcock Medical Center, Lebanon, NH
}

In the current era of evidence-based medicine, clinical practice guidelines (CPGs), care pathways, and best practice protocols are ubiquitous. CPGs are systematically developed statements, designed to assist clinicians and patients with decisions regarding appropriate care. ${ }^{1}$ Public health agencies and professional societies tasked with creating these guidelines are faced with the challenge of providing comprehensive, specific, understandable, and clinically usable recommendations. Underlying the tremendous effort of creating CPGs is the assumption that once the guideline is in place, it will be used to guide care.

In this issue of Annals of Surgical Oncology, Varey and colleagues present a large, population-based, cross-sectional study evaluating adherence to the Australian Melanoma Management Guidelines. ${ }^{2}$ The authors note only $35 \%$ of wide local excisions (WLEs) were performed with margins concordant with the guidelines; $45 \%$ of WLEs had more aggressive margins than recommended and the remainder were excised with inadequate margins. While nearly two-thirds of patients received guidelinediscordant care, implications on rates of recurrence and disease-related morbidity/mortality were not examined in this study and are unknown. To place these findings in a larger context, we must understand why the guidelines are not being followed. Physician knowledge, behavior, and attitudes, as well as a guideline's underlying 'trustworthiness' have all been identified as barriers to the uptake of CPG recommendations. ${ }^{3,4}$

(C) Society of Surgical Oncology 2017

First Received: 13 April 2017;

Published Online: 22 May 2017

S. L. Wong, MD, MS

e-mail: Sandra.L.Wong@hitchcock.org

\section{PHYSICIAN KNOWLEDGE}

It stands to reason that 'expert' physicians would be more familiar with the guidelines, and the data presented on physician volume support this assertion. Surgeons with high annual melanoma caseloads $(>30)$ were $27 \%$ more likely to perform a WLE concordant with the guideline than those with a lower caseload (1-5 cases annually). ${ }^{2}$ Broader dissemination of CGPs may bridge the gaps in care and improve care across all providers.

\section{PHYSICIAN BEHAVIOR}

Generally speaking, surgical margins for melanoma have become more conservative over time. While margins as wide as $5 \mathrm{~cm}$ were once commonplace, current guidelines from the National Comprehensive Cancer Network (NCCN), the European Society for Medical Oncology (ESMO), the Swiss Cancer League, and the Australian Cancer Network all recommend margins no greater than $2 \mathrm{~cm} .{ }^{5}$ Shifting to narrower excision margins represents a potential challenge to physicians who have experienced good oncological outcomes with wider margins. This inertia of prior experience may explain why nearly half of the excisions performed in this study had wider margins than recommended, and why Varey et al. ${ }^{2}$ found physicians aged 55-64 years were $32 \%$ less likely to adhere to guidelines compared with those aged under 45 years.

\section{PHYSICIAN ATTITUDES}

Convergence of clinical practice with care recommendations relies both on familiarity with, and positive attitudes toward, the CPG. Physicians interpreting the evidence on which a recommendation is based may come to a different conclusion and thereby consciously practice 
outside of the guideline. Even professional societies and government agencies responsible for generating CPGs have drawn conflicting recommendations using the same underlying evidence. ${ }^{6}$ Inconsistent recommendations across guidelines may also contribute to a laxity in adherence. This study noted overtreatment was more likely for in situ disease and thin melanoma. The 1999 Australian guidelines recommended $5 \mathrm{~mm}$ margins for in situ disease, while the contemporary NCCN guideline recommended margins up to $1 \mathrm{~cm}$. Indeed, some of what Varey and colleagues labeled as discordant care may be considered guideline-appropriate care if another CPG was used as the metric.

\section{TRUSTWORTHINESS OF GUIDELINES}

The Institute of Medicine (IOM) published eight standards for developing trustworthy CPGs (the so-called 'guidelines for guidelines'), including performing a rigorous systematic review, having a transparent process, and regularly updating the guidelines to reflect changes in professional consensus and availability of new evidence. While the 1999 CPG evaluated in this study predates the IOM publication, a retrospective evaluation highlights the lack of regular updating as a potential barrier to trust. This study evaluated cases diagnosed in 2006-2007, immediately prior to the release of the 2008 guideline. Over the span of 8 years, from release of the 1999 guideline to this evaluation of adherence, the body of evidence informing melanoma care evolved. Thus, the 1999 guideline may have been viewed as outdated, and perhaps some physicians were 'practicing ahead of the guideline', but in accordance with current evidence. To account for this, temporal trends in practice would have to be evaluated and examined in the context of evolving data.

Varey and colleagues have provided us with data to inform our understanding of adherence to CPGs for melanoma care. Specific recommendations that have a high degree of discordance require evaluation to ensure the evidence and its interpretation are transparent and credible. A better system to reconcile differences across various national guidelines must also be developed.

If trustworthy guidelines are in place and overall concordance is still poor, greater efforts must be undertaken to disseminate and implement the guidelines. To date, many public campaigns have successfully raised awareness of cancer prevention and screening strategies; it is time to move the dialogue of disease management into a similar forum for healthcare providers. Implementation, even in high-tech settings, demands ease of accessibility to highquality CPGs, and this may require better point-of-care decision support tools. Addressing physician attitudes and behaviors is critical, but attention must also be paid to emphasizing a link between guideline concordance and improved patient outcomes. Guidelines should guide practice and, ultimately, improve care.

\section{REFERENCES}

1. Lohr KN, Field MJ (eds). Clinical practice guidelines: directions for a new program. National Academies Press, Washington, DC; 1990.

2. Varey A, Madronio C, Cust A, Goumas C, Mann G, Armstrong B, et al. Poor adherence to national clinical management guidelines: a population-based, cross-sectional study of the surgical management of melanoma in New South Wales, Australia. Ann Surg Oncol. 2017. doi:10.1245/s10434-017-5890-7.

3. Cabana MD, Rand CS, Powe NR, Wu AW, Wilson MH, Abboud PA, et al. Why don't physicians follow clinical practice guidelines?: A framework for improvement. JAMA. 1999;282(15): 1458-65.

4. Ransohoff DF, Pignone M, Sox HC. How to decide whether a clinical practice guideline is trustworthy. JAMA. 2013;309(2): 139-40.

5. Ethun CG, Delman KA. The importance of surgical margins in melanoma. J Surg Oncol. 2016;113:339-45.

6. Abdelsattar ZM, Reames BN, Regenbogen SE, Hendren S, Wong SL. Critical evaluation of the scientific content in clinical practice guidelines. Cancer. 2015;121(5):783-9. 